\title{
There's a Measure for That!
}

\author{
Amir Roth ${ }^{\mathrm{a}, \mathrm{c}}$, David Goldwasser ${ }^{\mathrm{b}}$, and Andrew Parker ${ }^{\mathrm{b}}$ \\ ${ }^{a}$ Department of Energy, Washington, DC USA 20585 \\ ${ }^{b}$ National Renewable Energy Laboratory, Golden, CO USA 80406 \\ ${ }^{\circ}$ Corresponding author \\ amir.roth@ee.doe.gov, david.goldwasser@nrel.gov, andrew.parker@nrel.gov
}

\begin{abstract}
The OpenStudio software development kit has played a significant role in the adoption of the EnergyPlus whole building energy modeling engine and in the development and launch of new applications that use EnergyPlus for a variety of purposes, from design to auditing to code compliance and management of large portfolios. One of the most powerful features of the OpenStudio platform is Measures, a scripting facility similar to Excel's Visual Basic macros. Measures can be used to apply energy conservation measures to models - hence the name - to create reports and visualizations, and even to sew together custom workflows. Measures automate tedious tasks increasing modeler productivity and reducing error. Measures have also become a currency in the OpenStudio tools ecosystem, a way to codify knowledge and protocol and transfer it from one modeler to another, either within an organization or within the global modeling community. This paper describes some of the many applications of Measures.
\end{abstract}

\section{Introduction}

In current practice, common building energy modeling (BEM) tasks are codified and transferred via either documents-which every new modeler must interpret and implement in their chosen workflow-or via ad hoc observation and apprenticeship. These knowledge transfer channels have low bandwidth, are prone to human judgement and error and generally contribute to the wide variation in modeling practice and results observed across the industry. The US Department of Energy's (DOE) OpenStudio platform has a scripting facility called "Measures" that is transforming the way in which modeling tasks are performed and in which modeling knowledge is codified and shared.

OpenStudio is a strategic component in the DOE's efforts to increase the effective use of advanced BEM in building design and operation. The OpenStudio software development kit (SDK) is a library that maintains a detailed representation of the building energy model and the results of the simulation and provides functions-importing 3D geometry, creating standard HVAC systems, exporting models to EnergyPlus for energy analysis and to Radiance for lighting analysis, running simulations-that are common to many BEM applications. The SDK dramatically reduces the effort of BEM application development as the OpenStudio graphical application-developed in about four months-demonstrates. Over the past several years, public and private organizations have leveraged OpenStudio to deliver new applications and services, including DOE's Commercial Asset Score rating tool, Xcel Energy's EDAPT energy design-assistance project management portal, and the California Energy Commission's CBECCCom code-compliance application.

OpenStudio Measures are enabled by a combination of three features. First, the SDK provides direct access to OpenStudio's internal model of the building and the results of the simulation. Second, SDK 
functions can be invoked from interpreted scripting languages like Ruby and C\#. Finally, OpenStudio can interpret and apply scripts at runtime. Together these create a scripting facility that is similar to Microsoft Excel's Visual Basic macros. Just as Visual Basic has made Microsoft Excel a powerful and ubiquitous tool, Measures are having a similar impact on OpenStudio and its users. A modeler can encapsulate a specific procedure in a Measure and share that Measure with other modelers who can then apply it to their own models. Measures allow modeling procedures to be codified and shared in a consistent way that eliminates much of the human error inherent in document interpretation and data transcription. By improving modeling consistency and modeler productivity, Measures have become the most powerful feature of the OpenStudio platform and the core of its value proposition.

The original use of Measures_and the origin of the name "Measures"-is the transformation of an existing model representing the application of energy conservation measures (ECMs). However, Measures are as flexible as the underlying model representation and the scripting language and have found new uses and applications. The public repository Building Components Library (http://bcl.nrel.gov/) already contains over 190 Measures, and more are being added every week, by both the OpenStudio team and by users. The rest of the paper describes the various types of Measures and the applications they support.

\section{Model Measures}

Model Measures take an OpenStudio model as input and produce a transformed OpenStudio model as output. This structure aligns with the concept of ECMs, and many model Measures are used as ECMs. Model Measures can be simple "search-and-replace" routines, e.g., "replace exterior window construction A with construction B". However, with access to the full OpenStudio object model, they can also be arbitrarily complex. Full model access allows Measures to apply transformations selectively and surgically, e.g., "remove exterior window on facades oriented between degrees $X$ and $Y "$ ". More generally, it supports Measures that perform different transformations in different contexts as well as multiple coordinated transformations.

\section{Example: Simplistic Daylighting Package}

Figure 1 shows a Measure that applies a simplistic Daylighting ECM. To exploit Daylighting in the northern hemisphere, fenestration is usually removed or minimized on East and West facing facades and shaded on the South facade to minimize solar heat gain and glare. The top shows the Measure code, the bottom shows a simple box model before and after Measure application.

The Measure is written in the programming language Ruby. The run function is invoked when the measure executes. Statements beginning with \# are "comments" and are not part of the code, rather they are inline documentation. The Measure iterates through all sub-surfaces in the model-in OpenStudio walls are surfaces and wall openings including windows are sub-surfaces. It tests each sub-surface to ensure that it is an exterior window, then retrieves the azimuth and performs a specific action based on the azimuth value. North facing windows-azimuth between 315 and 45-are untouched. East and West facing windows are removed. South facing windows are fitted with an overhang. The high-level method addOverhangByProjectionFactor is provided by the OpenStudio SDK.

Those who are familiar with EnergyPlus and its input format (IDF) will notice how compact this code is relative to the EnergyPlus objects it manipulates. EnergyPlus fenestration sub-surface objects are 22 fields long. Removing a sub-surface object is not a simple one-line modification to an IDF file. Neither is creating an overhang surface given a window sub-surface object. This economy is provided by the OpenStudio SDK and its abstractions. 


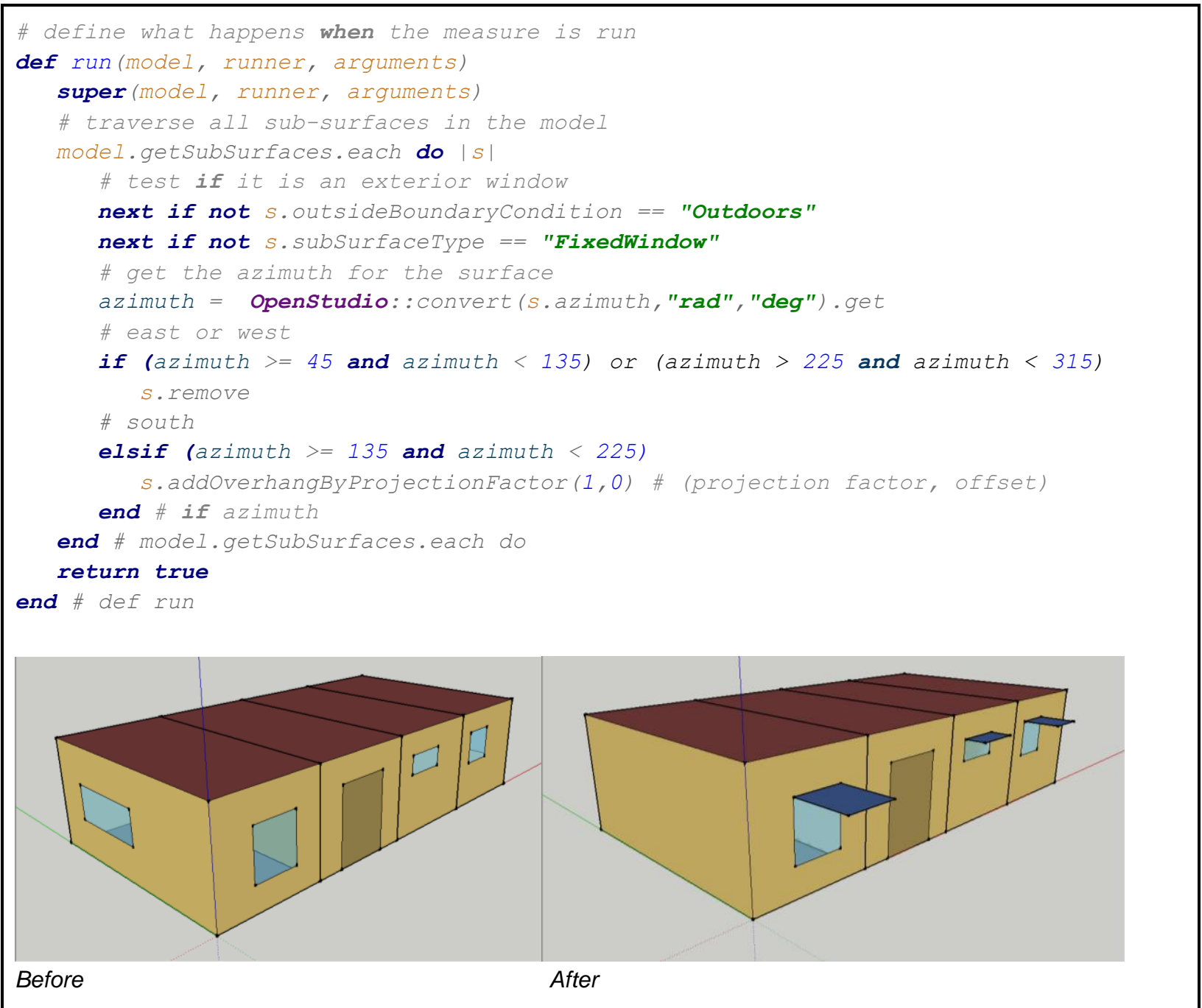

Figure 1. Simplistic Daylighting Measure. Top: measure code. Bottom: before-and-after illustration of measure.

\section{Example: AEDG K-12 School Daylighting Package}

The example Measure above is a highly simplified version of one of the most illustrative and visually impressive model Measures—the ASHRAE Advanced Energy Design Guide (AEDG) "K-12 School Daylighting Package" Measure. This Measure coordinates six different transformations. Like the simplistic Measure it: i) reduces or eliminates glazing on east and west facing facades, and ii) adds shading on southern facades to reduce glare and heat gain from direct solar beams-although it does this in a more robust and surgical way. It also: iii) reconfigures glazing on north and south facing facades to maximize daylight harvesting while minimizing glazing area, iv) properly uses clear and translucent glass, and light shelves to maximize the depth of daylight penetration $v$ ) adds and configures skylights in deep spaces to improve daylight penetration, and vi) adds, places, and configures daylight and occupancy sensors and electric lighting and blind controls. This Measure is applied properly to buildings of different geometries and space type allocations. The code is too long to list here but Figure 2 shows a "before" and "after" of the Measure applied to the DOE prototype secondary school model. 


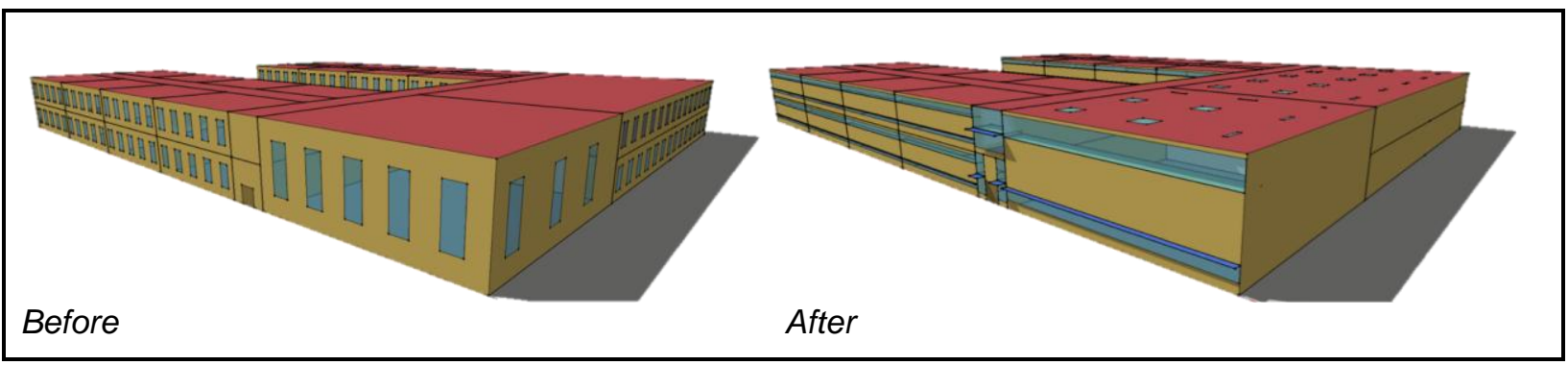

Figure 2. AEDG K-12 School Before and After Daylighting Package

view this measure on $B C L$

An important aspect of the daylighting Measure-and of many other Measures-is the use of standard space type descriptors to implement different ECMs in different areas of a building. For instance, skylights are useful in large deep spaces, but they are not appropriate for all such spaces-they work well in gymnasiums and cafeterias but not in auditoriums. In this Measure, space-type determines whether, where, and how much view glazing, daylight glazing, and skylights are included, and also informs the control scheme. The daylighting Measure is a good example of both the flexibility of Measures and the process of translating and codifying detailed, context-specific guidance-in this case from the ASHRAE AEDG.

\section{Example: Set Gas Burner Efficiency}

Model Measures can manipulate any part of a model, from geometry and constructions to HVAC systems. As a an additional example, Figure 3 shows a Model Measure that sets the gas burner efficiency on burners in all air loops to a user specified value. This example illustrates an additional feature of Measures, the ability to capture user arguments. Arguments allows a single Measure to implement a family of related ECMs by accepting different arguments. Arguments can be simple like an efficiency value or as complex as an entire component description. The arguments function is used by OpenStudio applications to query the user for arguments before passing them to the measure. Another feature of Measures is to test for conditions and send warnings, errors, and informative messages "up" to the user or calling application to inform them about the interaction of the Measure with the model. The method registerInfo on the runner object is used for this purpose.

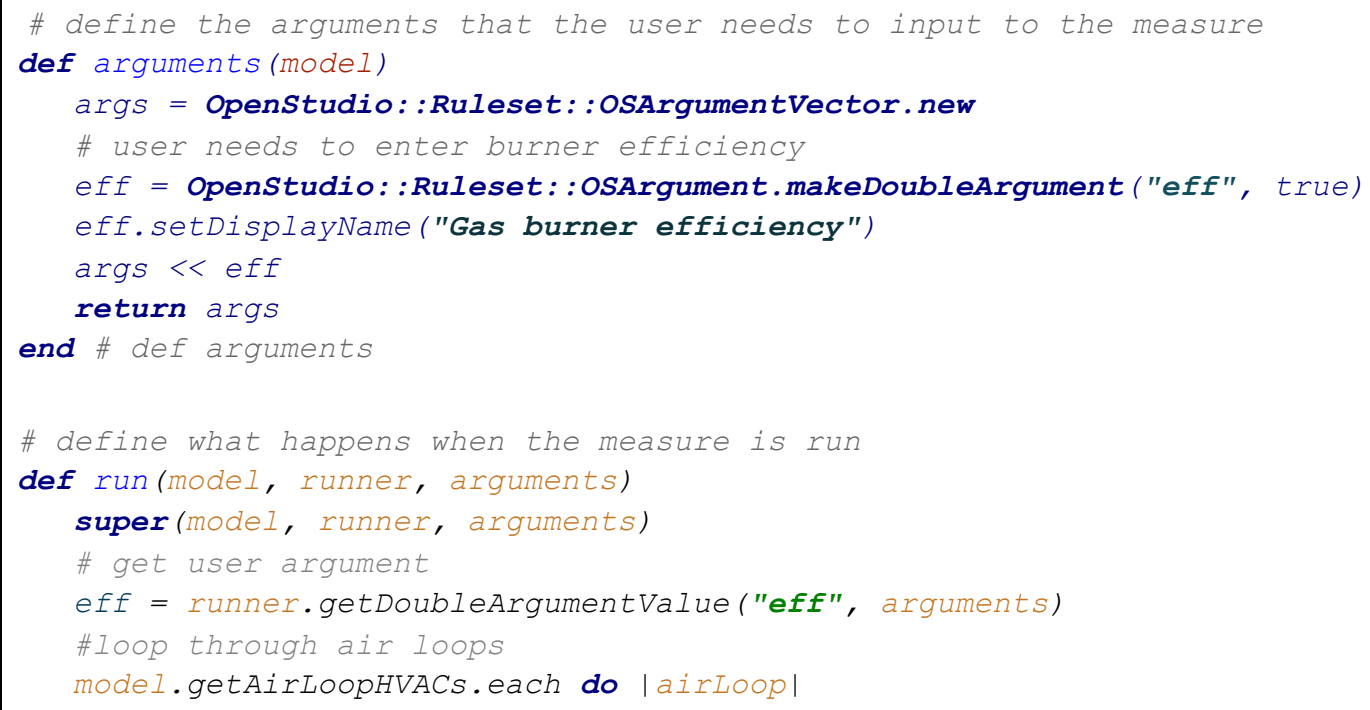




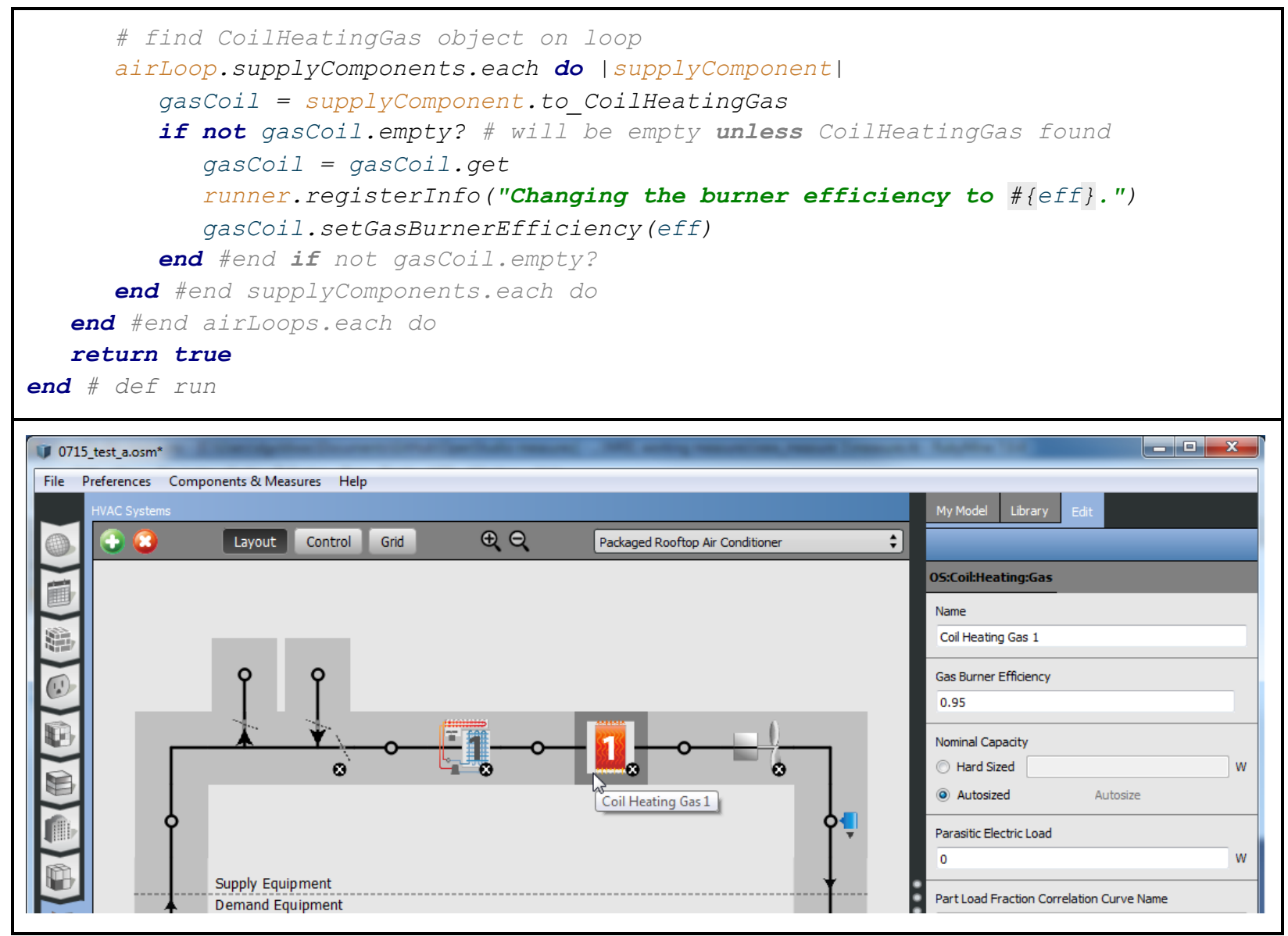

Figure 3. Set Gas Burner Efficiency Measure. Top: measure code. Bottom: measure result.

view this measure on $B C L$

\subsection{Model Measure Composition, Parametric Analysis, and Optimization}

The model-in/model-out paradigm allows Model Measures to be inserted seamlessly into any workflow. Where a model exists, a Model Measure can be used to transform the model before the workflow resumes. This property allows the same Model Measure to be used in different workflows, even ones for which it was not initially intended. A powerful special case of this characteristic is that Model Measures can be composed or chained to produce the combined effect of multiple transformations. When Model Measures correspond to ECMs, the effect is that of ECM packages.

The ability to apply Model Measures programmatically and systematically facilitates parametric analysis and design optimization. OpenStudio supports simple parametric analysis via the Parametric Analysis Tool (PAT) which is shown in Figure 4. In PAT, a user specifies a base model, and a set of Model Measures. PAT automatically creates the single-measure design alternatives by applying the Measures in the proper combinations, runs the resulting models, and displays the results for energy use, simple payback, and other metrics. 


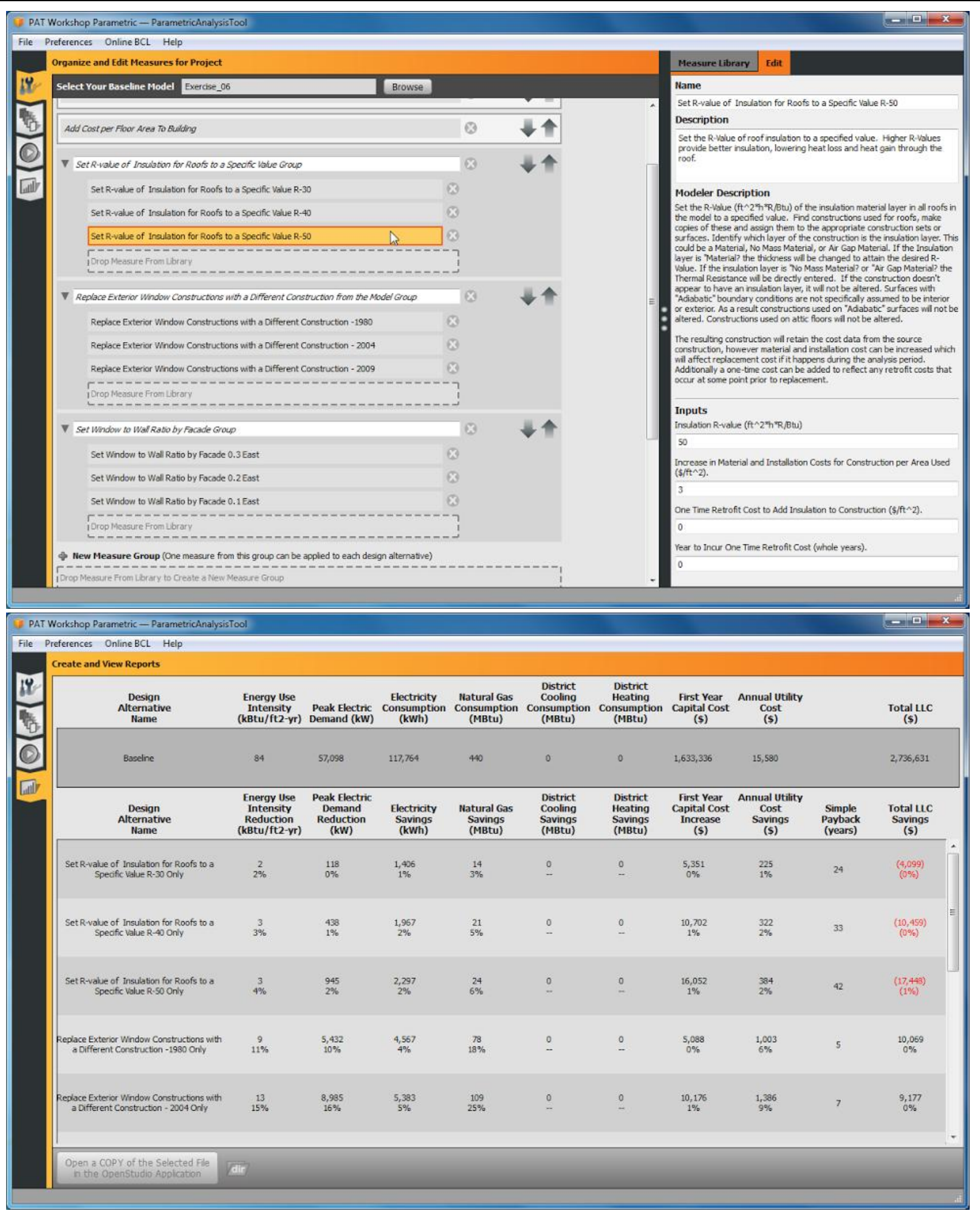

Figure 4. Parametric Analysis Tool. Top: PAT Measure selection screen. Bottom: PAT results for different Measures. 
For analysis of many design alternatives that would take too long using local computing resources, OpenStudio leverages the cloud. The economics of cloud computing are now such that large scale analysis comprising hundreds of thousands of simulations can be performed in a few hours for several thousand dollars. The time and cost are trivial compared to the resources that would be required to do this manually and locally. OpenStudio Server is a pre-configured "machine image" that can be deployed to the Amazon EC2 service. Users can launch instances of OpenStudio using their EC2 accounts, upload baseline models, Model Measures, and specifications for measure combinations and then download results for analysis and visualization. Several organizations including DOE and National Grid, a utility operating in several northeast states, are using this service to support their programs with large scale analyses at low cost.

Figure 5 shows the inputs and results of an OpenStudio Server large scale analysis. To facilitate setup, OpenStudio Server accepts specifications in the form of a spreadsheet. Each group of rows represents a Measure, e.g., "Create Aspect Ratio with Rotation", "Window to Wall Ratio South", etc. The top row in each group contains information about the Measure itself; the remaining rows contain information about inputs to the Measure. An argument input is held constant over the entire analysis, e.g., for the "Create Aspect Ration with Rotation" Measure, "Total Building Floor Area" is held constant at $20000 \mathrm{ft}^{2}$. A variable input is varied across the analysis as "Number of Floors" is for the same Measure. A variable can be either continuous or discrete.

To ease inspection and manipulation of large batches of simulation output, it returns selected results as an $\mathrm{R}$ data frame. These results can be manipulated programmatically or viewed interactively using active widgets like a parallel coordinate plot (PCP) shown at the bottom of the figure. Each vertical axis represents an input parameter or output value. Each simulation is represented by a "horizontal" multi-line. Sliders on each axis allows the user to filter the data-set interactively with filtered results grayed out. One insight apparent from the PCP in the Figure is that most of the low-EUI configurations correspond to twostory buildings.

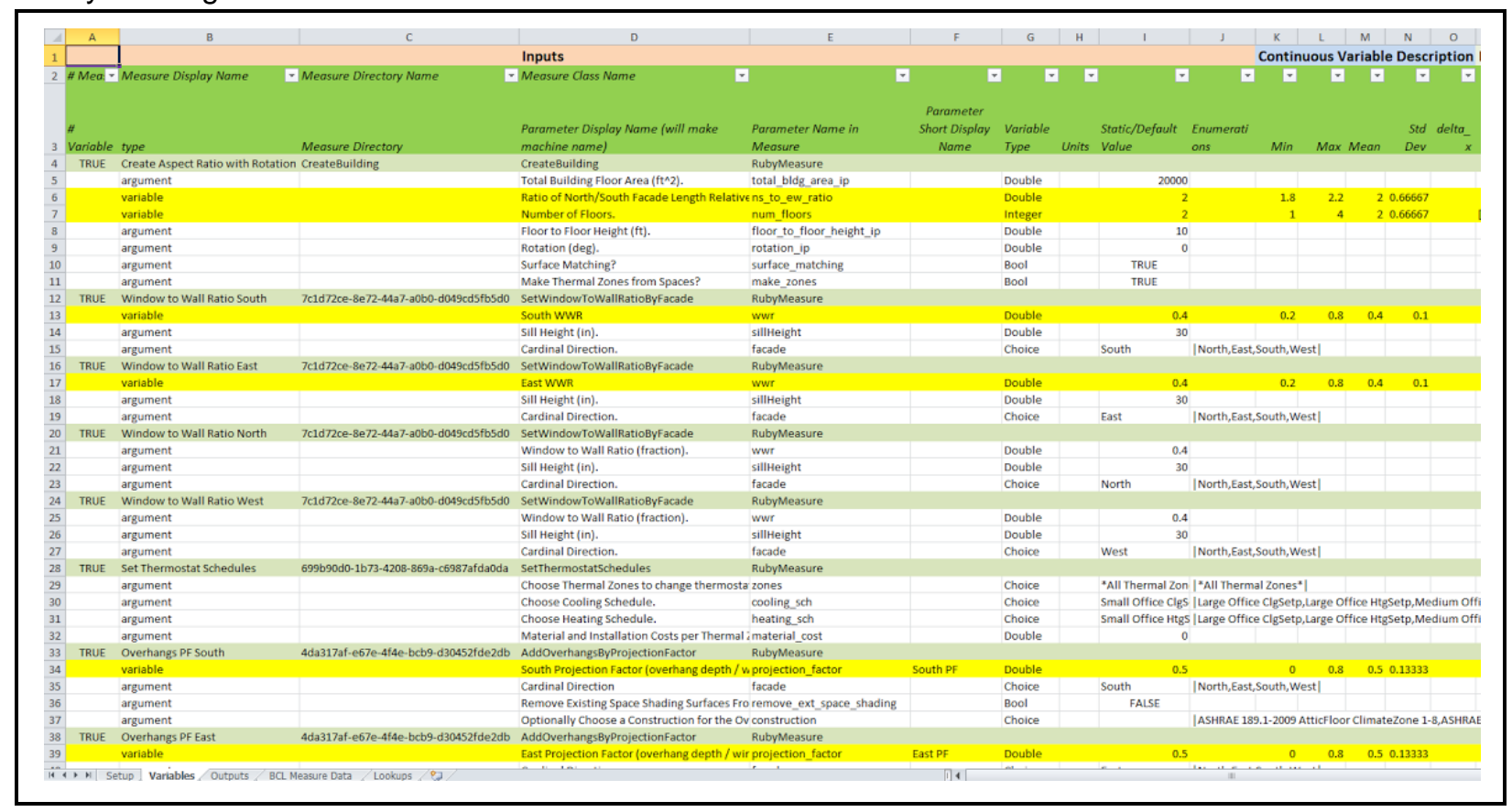




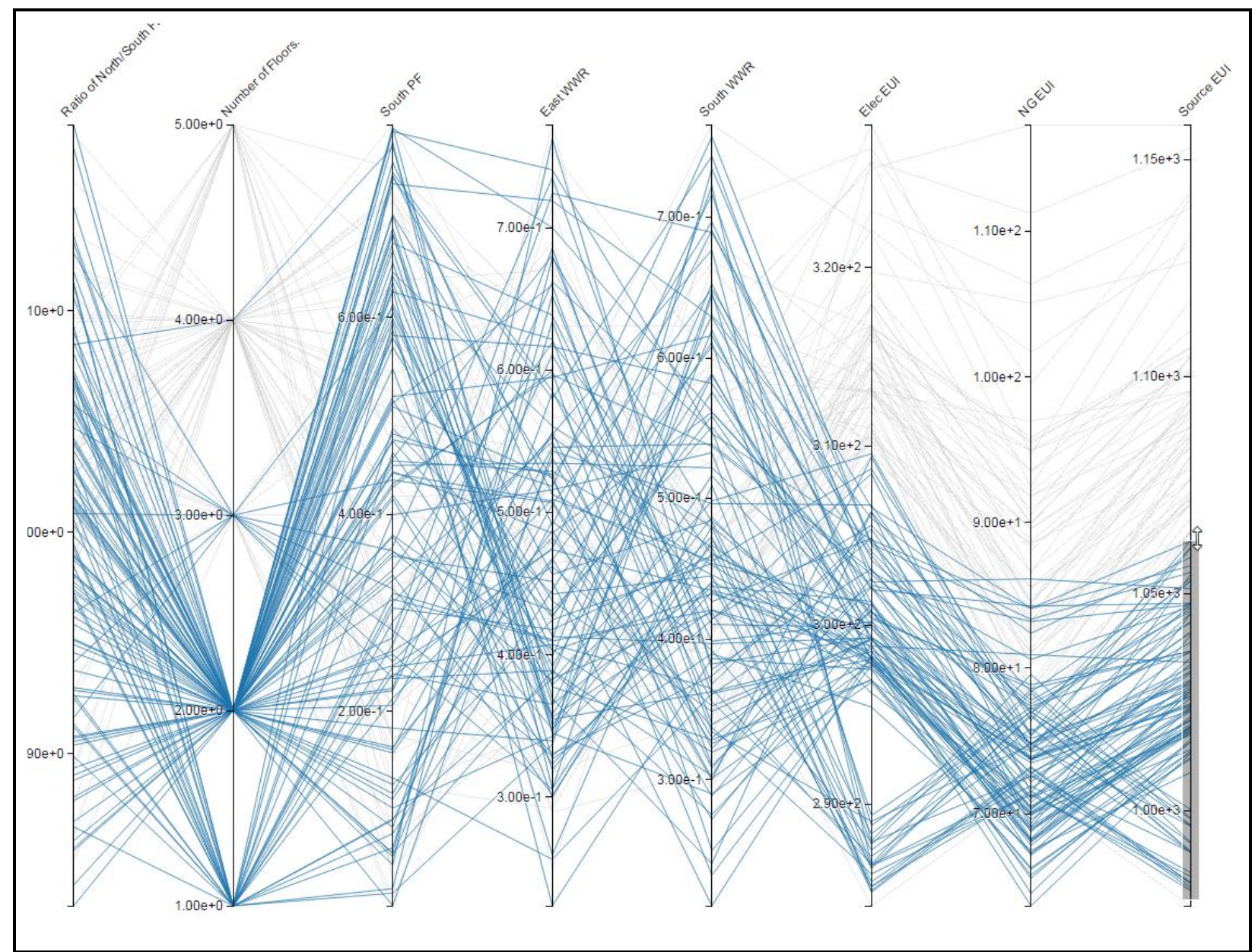

Figure 5. OpenStudio Server. Top: Analysis specification spreadsheet. Bottom: Analysis results presented using an interactive parallel coordinate plot widget.

In setting up a parametric analysis—large or small—it is important to remember that OpenStudio Measures are not explicitly aware of other Measures that have already been applied to the model. The user must take care to specify Measures in the proper application order, e.g., a Measure that creates fenestration should always be applied after a Measure that creates basic geometry. In general, although Measures that manipulate different parts of the model are commutative-an HVAC Measure can be applied before a plug-load Measure or vice versa-Measures sometimes have subtle interactions that make application order significant. Measure application order should generally be carefully considered in any workflow. To generate a sample model, OpenStudio Server applies Model Measures in the order in which they appear in the spreadsheet, e.g., "Window to Wall Ratio" Measures are applied after overall building aspect ratio and number of floors are set.

\subsection{Procedural Modeling and Model Measures As Database Indices}

Model Measures can be used to build up complete models from scratch, starting with an empty model and successively creating parameterized geometry, assigning space load and occupancy schedules, and articulating and mechanical systems and linking them with zones. This "procedural modeling" is useful in large scale analysis. It also has another subtle and powerful advantage. Model Measures are a compact and logical way to store, organize, and index large collections of models. An EnergyPlus model has tens 
of thousands of individual data elements. Warehousing a set of models requires a large, complex database with tens of thousands of attributes. However, an EnergyPlus model created procedurally using successive application of Model Measures can be completely described, stored, and indexed using a few dozen attributes - the identifiers of the Model Measures used to create the model along with any arguments required by those Measures. "Procedural" models can be stored in a small database that is organized logically and can be searched quickly and easily. If the results encoded in the database ever need to be updated or expanded, the compact specifications are expanded into full models and resimulated. OpenStudio exploits compact specification and ease of organization in a procedural modeling database framework called DEnCity.

\subsection{Uncertainty and Sensitivity Measures and Model Input Calibration}

Model Measures can also transform models in ways that don't correspond to ECMs. For instance, they can implement uncertainty or sensitivity perturbations-composing these yields uncertainty or sensitivity analysis.

Figure 6 shows an uncertainty Model Measure, which builds off of the "Set Gas Burner Efficiency" Model Measure from Figure 3. Rather than taking a new efficiency as an argument, this measure takes an uncertainty distribution - defined as a relative mean, standard deviation, minimum, and maximum-and uses it to generate a multiplier which is then applied to the existing efficiencies of all gas burners. By moving the line of code that generates the multiplier by "calling" the distribution object into the inner loop, this code could be modified to generate a different uncertainty multiplier for each gas burner. This Model Measure is indicative, it is not an existing Measure that can be downloaded from the Building Component Library. The uncertainty Measure framework is still under development.

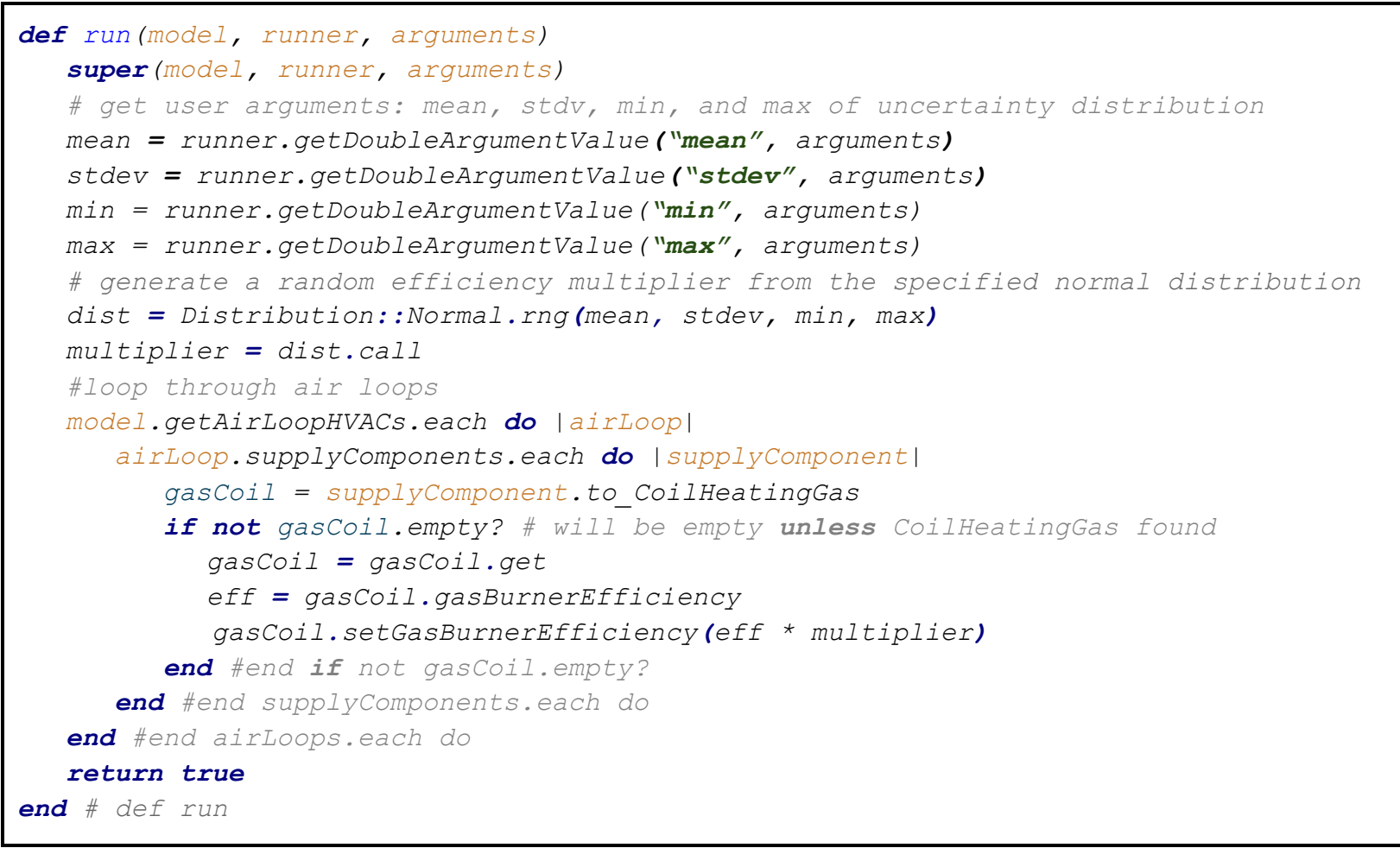

Figure 6. Indicative Uncertainty Model Measure 
Uncertainty measures can be combined with an objective function that minimizes the difference between simulated energy use and measured energy use from monthly utility bills to implement model input calibration.

\section{Reporting Measures}

Model Measures query and transform models. Reporting Measures query input models and query and transform simulation results. Reporting Measures can be used to create custom reports, take-offs and visualizations, and to perform sanity and quality-assurance checks.

\section{Example: Space Type Summary Report}

Figure 6 shows a library method that performs a space-type "take-off" to produce a breakdown table and chart. This library method is invoked from a larger Reporting Measure to create a detailed report that covers all aspects of the model.








Figure 7. Space Type Summary Reporting Measure. Top: measure code. Bottom: output. view this measure on $B C L$

\section{Example: Zone Conditions, Humidity Report}

Figure 7 shows a results visualization method that feeds a Reporting Measure. Rather than querying the modeling, this measure queries the simulation results which are stored in the top-level sqlFile object. This Reporting Measure queries the time-series data in the results file to create a tabular histogram of hourly relative humidity ranges for zone. The resulting table shows the number of annual hours every zone spends in a given humidity range. The bins are colored by annual hour thresholds to make it easy to identify the most prevalent conditions in every zone.






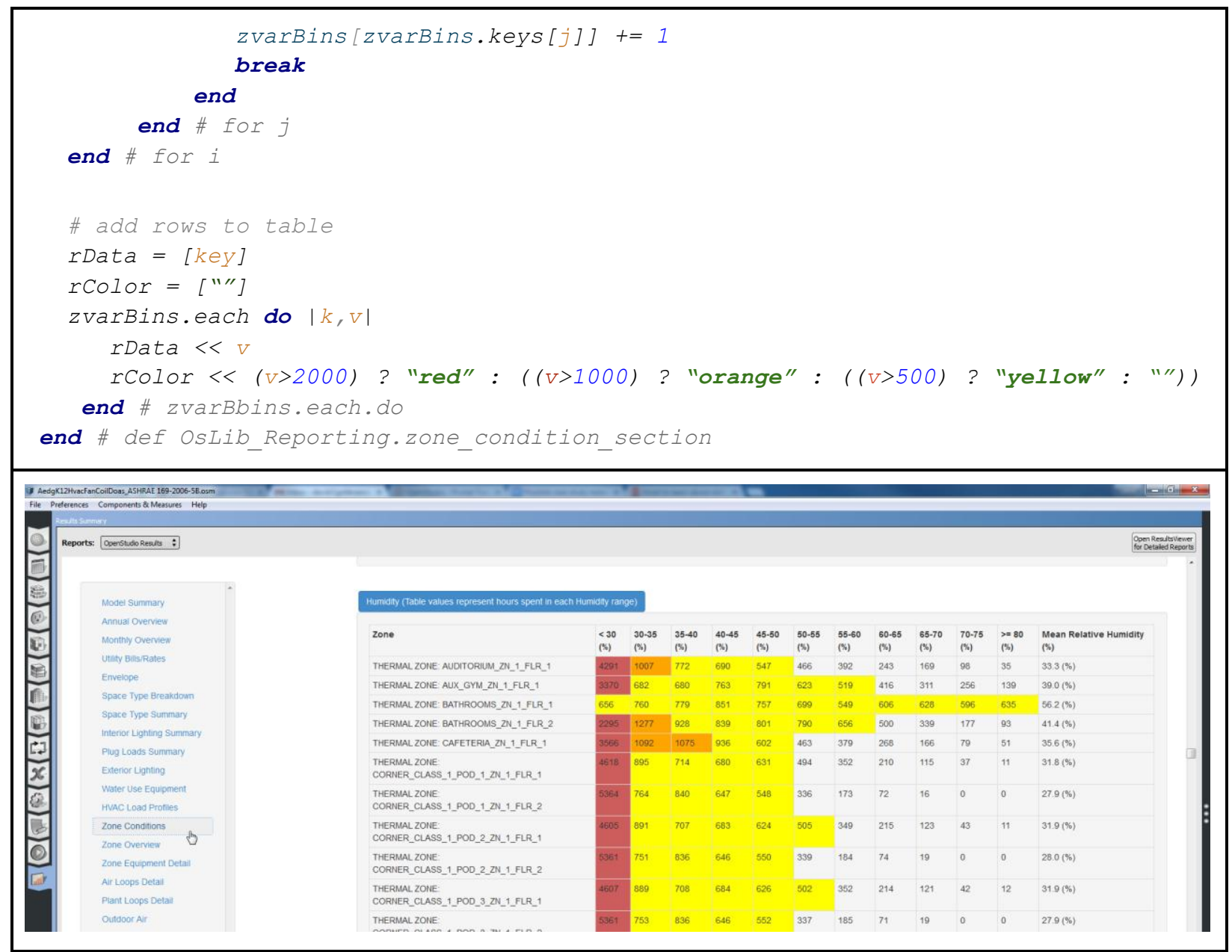

Figure 8. Zone Humidity Histogram Reporting Measure. Top: measure code. Bottom: output.

view this measure on $B C L$

\section{Example: View Model and View Data}

Two new Reporting Measures currently under development export OpenStudio model geometry to the vA3C JSON format (http://va3c.github.io/). vA3C allows a user to visualize a building model in any web browser using Three.js (http://threejs.org/), a cross-browser JavaScript library for animated 3D graphics. The exported model can be viewed interactively, i.e., panned, zoomed, orbited and even filtered, with parts selectively faded out to focus on interesting parts of the model. The View Model measure can be used at any point in the workflow and offers a variety of color-rendering modes based on surface and space attributes such as surface type, surface boundary condition, surface construction, space type, and thermal zone. Figure 9 shows an example application of the View Model measure with surfaces rendered by type. 


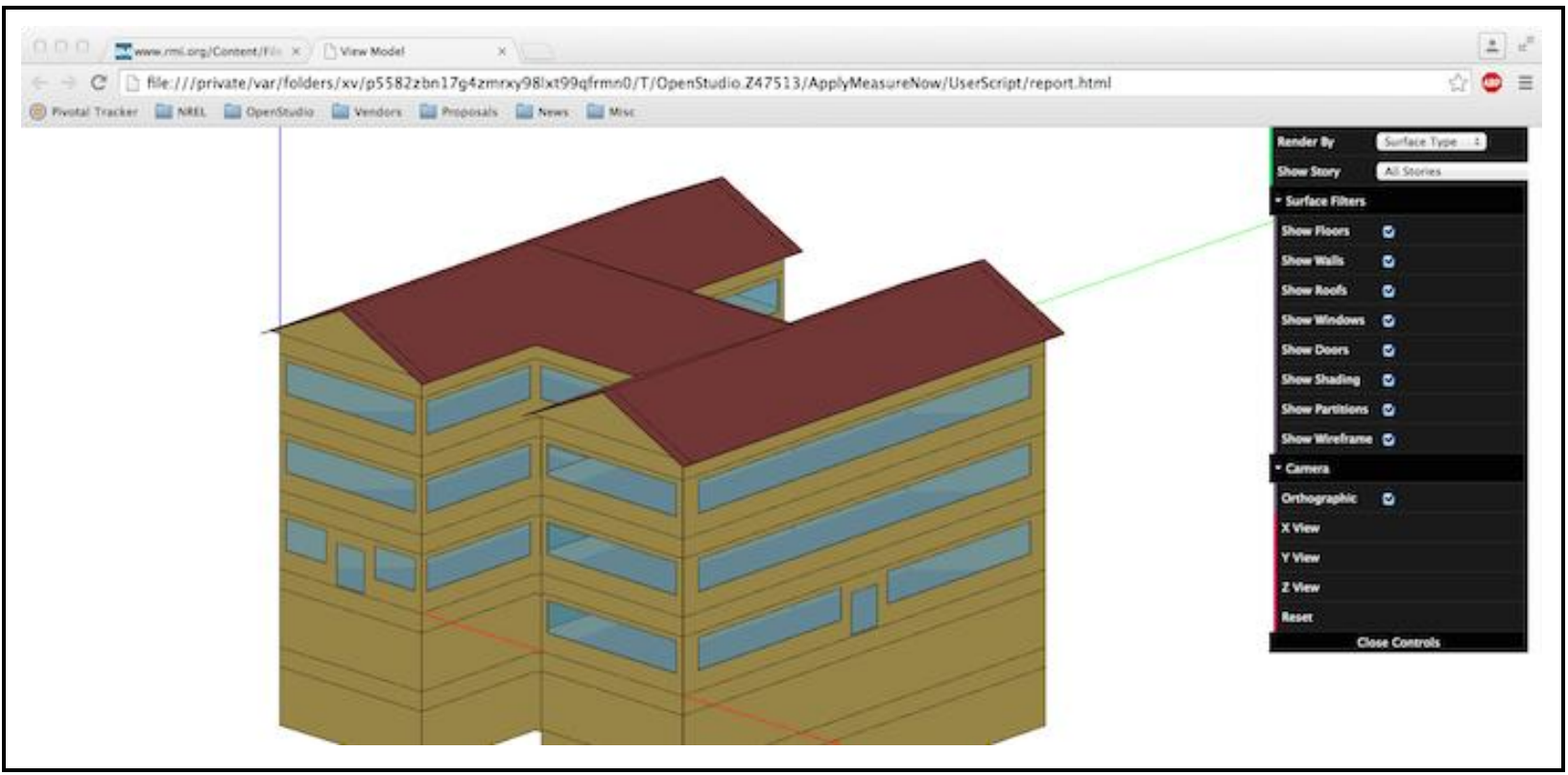

Figure 9. View Model Reporting Measure.

view this measure on $B C L$

The View Data measure is a Reporting Measure that can only be used as part of a runtime workflow. View Data extends View Model by allowing the user to render surfaces or zones by associated timeseries variables like temperature. The user can "play" the time series, rendering colors changing with the underlying data. Measures allow OpenStudio to exploit this new and powerful interactive visualization environment.

\section{Workflow Measures}

Workflow Measures automate workflows that chain multiple simulations or analyses together. Workflow Measures generally perform data import/export, processing the output of one tool into input for another. They can also orchestrate and synchronize the simulations themselves. Workflow Measures can link tools like EnergyPlus and Radiance that are "native" to OpenStudio; OpenStudio explicitly accounts for these tools in its internal representation, sometimes including objects and information that is specific to those tools, it supports import from and export to these tools as core functions, and sometimes even includes them in its own installer.

One Radiance-to-EnergyPlus Workflow Measure exports the OpenStudio model to Radiance format, runs Radiance to calculate detailed daylighting metrics, and then uses these to create electric lighting and shade control schedules for a subsequent EnergyPlus simulation. Another example uses Radiance to calculate custom "view factors" for surfaces within a zone, which are then used by EnergyPlus in interior radiant exchange calculations. Although EnergyPlus has intrinsic modules for calculating lighting metrics and view factors, Radiance can perform these calculations in a more robust and detailed fashion that may be needed in certain situations. A Workflow Measure is a lightweight alternative to reimplementing Radiance functionality within EnergyPlus or to coupling the programs at a tighter level that would be more invasive to both code bases.

Workflow Measures can also link OpenStudio analyses to external analysis. One example is a Workflow Measure that links GLHEPro to EnergyPlus to size and configure ground heat-exchange loops. Several 
organizations are creating custom Workflow Measures that connect EnergyPlus and Radiance to the specific suite of tools they use.

Access to the system allows Measures to encapsulate entire workflows. Measures are not limited to executing either before or after a simulation. A Measure may encapsulate a simulation, i.e., invoke the simulation itself and execute both before and after it. In essence, a Measure can use a simulation to inform its transformations and queries. This is a powerful automation paradigm.

One exciting Workflow Measure that is currently under development at Natural Resources-Canada imports data from DOE-2.2 input (.inp) files. The measure currently imports geometry only. But schedules, constructions, space types, and zones will be imported soon. And HVAC system descriptions will eventually be imported also. The model shown in Figure 8 was imported from a DOE-2.2 input file.

\section{The Measure Ecosystem}

In addition to their functional code "contents", Measures obey invariants and are tagged with attributes that are used by the OpenStudio tools ecosystem to simplify Measure writing and use.

As shown in Figure 3 (the "Set Gas Burner Efficiency" Measure), Measures consume arguments in structured ways and provide structured support functions for capturing them from users. Graphical User Interfaces (GUIs) use these to display Measures to users and allow them to select and specify argument values. These relieve GUI developers from writing Measure-specific GUI code and relieve the Measure developer from writing any GUI code at all. Figure 3 also showed how Measures can use OpenStudio hooks to trigger checks before and after Measure execution and to log informative, warning, and error messages.

Being written in general purpose scripting languages, Measures can make use of system resources beyond the OpenStudio model. Existing Measures regularly make use of Excel spreadsheets and JSON databases to store model data. This allows users to customize Measures without editing the code files. Measures can also use the BCL as a data source-in addition to Measures, the BCL contains over 47,000 simulation objects corresponding to constructions, equipment, loads, and schedules.

Measures are also associated with meta-data such as title, description, version, type (e.g., Model or Reporting) and whether Measure execution requires simulation results or not. This meta-data allows applications to make Measures available at the right points in the workflow and to support Measure filtering, search, and in-place documentation-this is shown in Figure 4. Meta-data also includes provenance (i.e., authorship and ownership) information allows organizations to control their visibility and distribution.

\section{Alternatives, Current Limitations and Future Work}

OpenStudio Measures are a powerful way of working with EnergyPlus models and simulation results.

However, there are alternatives including the native EnergyPlus objects that support parametric analysis, the EP-Macro utility that is distributed with EnergyPlus, and Eppy, an EnergyPlus input/output file reader/writer library written in python. OpenStudio Measures differ from these various alternatives in several ways. Unlike parametric objects and EP-Macro, OpenStudio Measures are written in a general- 
purpose language and can use the full expressive power of that language. They can also access EnergyPlus result files. Written in the general-purpose language python, The Eppy library is similar in concept to the OpenStudio SDK, and it is likely that many simple OpenStudio Measures can be recreated using Eppy. However, the OpenStudio SDK is significantly more developed and mature than Eppy and provides higher levels of abstraction and functionality. It is not clear whether Eppy will continue to add mass and function or remain a lighter-weight solution, in terms of both capability but also packaging and overhead.

OpenStudio Measures do have limitations. One restriction is that Measures only provide "first-class" access to aspects of the model that have native OpenStudio representations. As of this writing, OpenStudio has native representations of about $70 \%$ of the most commonly used EnergyPlus objects. EnergyPlus-only objects can be manipulated via "IDF Measures" which manipulate the EnergyPlus IDF input file after the model has been exported. IDF Measures are more cumbersome and can only be invoked after IDF export, at which point no "native" Model Measures can be invoked. IDF Measures provide a stop-gap solution for objects not yet included in the OpenStudio object model and their use should diminish over time as coverage expands. The most significant EnergyPlus feature not currently covered by native OpenStudio measures is the Energy Management System (EMS) user defined control feature.

Measures currently cannot interact with the user during Measure execution. Measure arguments are also fixed at the beginning of Measure execution-a Measure cannot ask for different input or change input default values based on inputs already provided by the user. Finally, Measures cannot interact with a running EnergyPlus simulation, as is possible via the Building Control Virtual Testbed (BCVTB).

As Measures have proven themselves more useful, DOE and NREL are increasing the investment in Measure infrastructure. Near term plans include the addition of automated regression testing to the $B C L$, ensuring that additions to the OpenStudio API or changes to its implementation do not break existing Measures.

\section{Conclusions: The Tao of Measures}

The computing industry has evolved by organizing itself into "horizontal" layers separated by fixed application programming interfaces (APIs), which are essentially service contracts. Layer A provides a set of abstractions and functions for use by Layer B above it. Layer B uses these to implement higher-level abstractions that serve Layer C. The fixed APIs allow layers to evolve largely independently of one another without knowledge of implementation of the layers below them, and it allow users to upgrade one layer at a time. In the general-purpose computing industry, Layer B is the operating system, which provides nice programming abstractions like files, threads, and graphical widgets on top of the raw hardware Layer A-which itself provides primitives like disk locations, instructions, and network ports-to facilitate the development of applications Layer C. The OpenStudio SDK is essentially an operating system for the BEM software stack. It builds on EnergyPlus and implements high-level abstractions that facilitate BEM application development.

Measures sit in a unique-and uniquely advantageous spot-in this ecosystem. In many ways, Measures resemble Layer $\mathrm{C}$ applications, and enjoy their benefits. They build on OpenStudio's easy-to-use highlevel abstractions and are written in scripting languages that are easier-to-program than $\mathrm{C}_{++}$which is used in both OpenStudio and many applications. Users can upgrade to newer SDK versions and expect their existing Measures to continue working - the OpenStudio SDK itself insulates client applications including Measures from EnergyPlus updates. And because Measures are not part of the SDK 
distribution, Measure developers are not subject the justifiably strict documentation, code-review, and testing requirements for SDK features. At the same time, Measures act like part of the (Layer B) operating system, they extend the functionality of the SDK and can be shared and used by any application including by other Measures.

Measures have the power and reach of core OpenStudio functions, but with the lightweight development requirements and accessibility of simple scripting. OpenStudio is an open-source project, so anyone may create a private version with custom core functionality. But there is no need to do so when Measures are just as powerful and the technical and organizational barriers to developing and using Measures are much lower than they are to core development.

Apple's iOS mobile operating system enabled rapid development and deployment of mobile applications via an online repository, the Apple Store. The flood of applications spawned the marketing slogan "There's an app for that!". OpenStudio has enabled rapid development and deployment of BEM applications via an online repository, the Building Component Library (BCL). The flood of Measures that has followed motivated the title of this paper.

\section{Acknowledgements}

The authors thank the editors and reviewers who contributed to improving this manuscript, the OpenStudio development team, and the many developers of Measures. OpenStudio is funded by the U.S. Department of Energy under an FFRDC (federally-funded research and development center) direct funding agreement with supplementary funding from outside organizations including the California Energy Commission and Xcel Energy of Colorado via CRADA (collaborative research and development agreement) and WFO (work for others) arrangments.

\section{References}

1. Brook, M., Criswell, S. "The BEE Software Collaborative: An Open-Source, Rule-Based Architecture for Building Energy Efficiency", Proceedings of the ACEEE Summer Study on Energy Efficiency in Buildings, Pacific Grove, California, August 2012.

2. Elling, J., Brackney, L., Parker, A., Long, N., "Energy Design Assistance Project Tracker (EDAPT) : A Web-Based Tool for Energy Design Assistance Program Management," Proceedings of the ACEEE Summer Study on Energy Efficiency in Buildings, Pacific Grove, California, August 2014.

3. Crawley, D., Winkelmann, F., Lawrie, L., Pedersen, C., "EnergyPlus: New Capabilities in a Whole-Building Energy Simulation Program", Proceedings of Seventh International IBPSA Conference, Rio de Janeiro, Brazil, 2001.

4. Fleming, K.; Long, N.; Swindler, A. (2012). "Building Component Library: An Online Repository to Facilitate Building Energy Model Creation"; NREL Report No. CP-5500-54710, August 2012.

5. Hale, E., Lisell, L., Goldwasser, D., Macumber, D., Dean, J., Metzger, I., Parker, A., Long, N., Ball, B., Schott, M., Weaver, E., Brackney, L., "Cloud-Based Model Calibration Using OpenStudio," eSim Conference, Ottawa Canada, May 2014.

6. Long, N., Ball, B., Brackney, L., Kruchten, D., Elling., J., Davis, O., "Leveraging OpenStudio's Application Programming Interfaces," International Building Performance Simulation Association Building Simulation 2013, Chambery, France, August 2013.

7. Macumber, D., Ball, B., Long. N., "A Graphical Tool for Cloud-Based Building Energy Simulation", Proceedings of ASHRAE IBPSA-USA Building Simulation Conference, Atlanta, Georgia, August 2014. 
8. NREL, "OpenStudio Measure Writing Guide", retrieved Sep. 9, 2015 from http://nrel.github.io/OpenStudio-user-documentation/reference/measure_writing_guide/.

9. Parker, A., Benne, K., Brackney, L., Hale, E., Macumber, D., Schott, M., Weaver, E., "Parametric Design Tool or Building Energy Analysis Workflows: Application to a Utility Design Assistance Incentive Program," Proceedings of the ACEEE Summer Study on Energy Efficiency in Buildings, Pacific Grove, California, August 2014.

10. Philip, S., "Eppy's Tutorial — Eppy 0.5 Documentation”, retrieved Sep. 9, 2015 from http://pythonhosted.org/eppy/.

11. Roth, A., Brook, M., Hale, E., Fleming, K., Long, N., "DEnCity: An Open Multi-Purpose Building Energy Simulation Database," Proceedings of the ACEEE Summer Study on Energy Efficiency in Buildings, Pacific Grove, California, August 2012.

12. Wang, N., Taylor, C., McCabe, M. "DOE Commercial Building Energy Asset Rating: Market Research and Program Direction", Proceedings of the ACEEE Summer Study on Energy Efficient Buildings, Pacific Grove, California, August 2012.

13. Wang, N., Gorrissen, W. "Commercial Building Energy Asset Score: Program Overview and Technical Protocol (Version 1.0)", Pacific Northwest National Laboratory Technical Report PNNL22045, December 2012.

14. Wetter, M., Haves, P. "A Modular Building Controls Virtual Testbed for Integrating Heterogeneous Systems", Proceedings of the Third IBPSA-USA Conference, Berkeley, California, August, 2008.

15. Zirnhelt, H. "Incorporating Uncertainty Analysis into Retrofit Analysis Using OpenStudio and EnergyPlus", Proceedings of ASHRAE IBPSA-USA Building Simulation Conference, Atlanta, Georgia, August 2014. 